\title{
Study of Error Establishment in Milling Machines with 5 Axes
}

\author{
Tsvetan Kaldashev \\ Technical university of Sofia \\ Sofia, Bulgaria \\ email: kaldashev.cvetan@abv.bg
}

\begin{abstract}
This report examines the accuracy of rotary die processing on a 5 axes machine and a special boltdisk system. Faults that affect the accuracy and their measurement and reduction within acceptable limits are analyzed. As a result of the measurement, a virtual model of the radial beating of the workpiece relative to the actual axis of rotation of the machine was developed.
\end{abstract}

Keywords - CNC, milling machine, error detection, 5 axis.

\section{INTRODUCTION}

When machining rotationally symmetrical details on 4 and 5 axis milling machines, setting is most often done in a chuck. As a result, there are set-up errors related to the radial and axial beating of the machine masses. These errors, as well as machine positioning errors, have a significant effect on the accuracy of processing [1], [2], [5]. With modern CNCs, different commands are available, with which some of these errors can be compensated. In the Fanuc CNC, the G54.2 [3] command is used, which sets the position of the workpiece set on a circular axis as a support compensation for the tool. Thus, when rotating the circular axis, the $\mathrm{CNC}$ automatically receives the current compensation of the device based on the angular displacement of the mass and creates a suitable coordinate system of the workpiece (figure1). Once the support compensation is established, the coordinate system of the workpiece is created dynamically regardless of the position of the rotary mass. The new position of the coordinate system of the workpiece is obtained by adding the fitting compensation to the initial set offset by a reference point of the machine.

For older CNCs, the offset of the set-up error is performed using devices where the radial beating error is measured with an indicator clock.

\author{
Petar Hadjiiski \\ Technical university of Sofia \\ Sofia, Bulgaria \\ email: phad@tu-sofia.bg
}

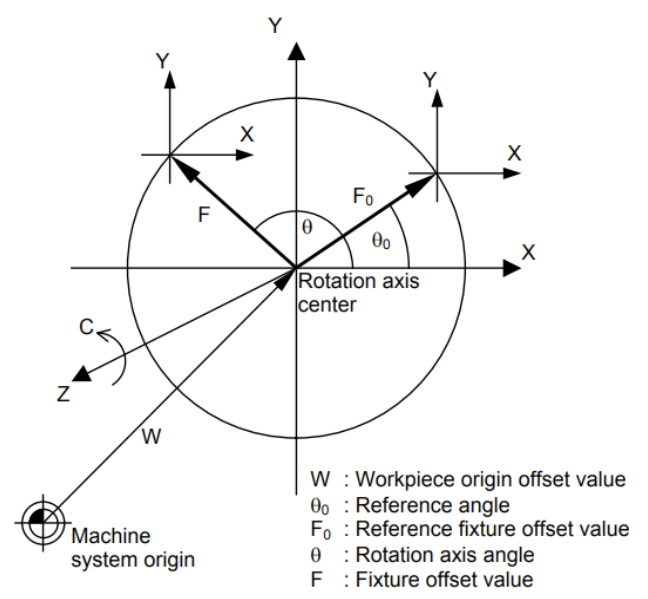

Fig. 1. Rotary table dynamic fixture offset

\section{STUDY OF ERROR ESTABLISHMENT WITH FIXTURE}

The workpiece alignment device consists a disk with a bolt system 1 and 2 (Figure 2) located on the flange of the chuck 3 at a central angle of 90 degree. The bolts 1 are used to secure the chuck to the rotating mass C. Bolts 2 are in contact with the mass of the machine, thereby pushing the chuck (rotating it around a point located at 180 degree relative to the bolt used for adjustment). In this way, the axis of the workpiece is displaced relatively to the axis of rotation of the mass $\mathrm{C}$, while the radial beating becomes as small as possible.

As an example will be discussed detail - knife for a rotary die for a sanitary absorbents. In these details, the width of the cutting blade (Figure 3 ), which is finally obtained by grinding the side surfaces 1 and 2 of the cutting contour [4], is of great importance. The mounting is made on the bearing and the front face of the shaft (Figure 4). 


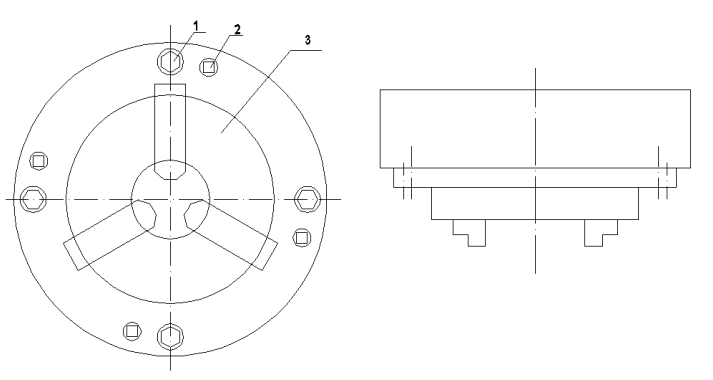

Fig. 2. Workpiece alignment tool

As a result, a base error arises due to the in organized base shifting. It appears because before the machine detail is found, it is grinded between the centers of a grinding machine on the cylindrical surface " $A$ " on which lies the cutting contour "B". Once a piece is found in the chuck of the machine, an error occurs due to the non-alignment of the axis of the workpiece 1-1 with the axis of rotation of the mass $\mathrm{C}$. On the other hand, there is a mass positioning error A -.

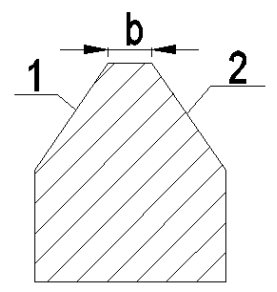

Fig. 3. Cutting width $\mathrm{b}$

As a result of the beating, the axis of the workpiece 1-1 makes a precession around the axis of the machine O-O. As a result, the radial and frontal rolling of the cylindrical surface "A", on which is located the cutting contour "B" having a trapezoidal section (Figure 3), has a significant impact on the precision of processing of the workpiece.

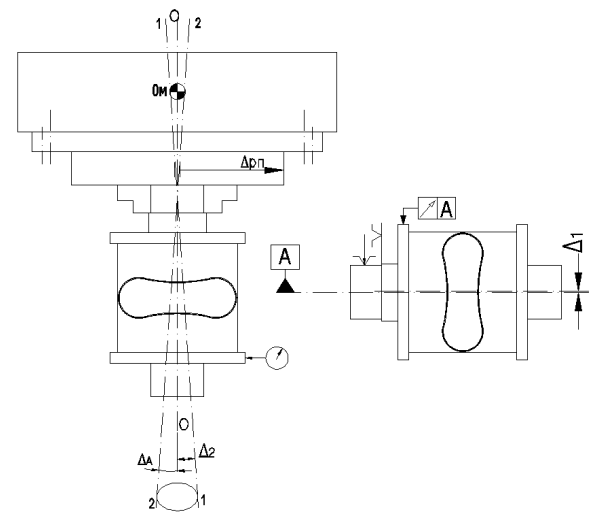

Fig. 4. Errors in establishing detail rotary die

The cutting capability of the contour sections is determined by the width $\mathrm{b}$ of the cutting track, and it must change for the time between two resharpenings in the bmin to bmax range. The maximum permissible width so that cuts are obtained is bmax and bmin is the minimum width allowed so that no breakage occurs and a resource is provided between two resharpenings. In order to reduce the impact of the settlement error caused by a shifting in the bases, it is necessary to work on a method to compensate it for a full turnover. After setting the machine detail, the radial beating is measured with an indicator clock set in the spindle of the machine. Measurement is carried out at A axis position of $90^{\circ}$ (Figure 5).

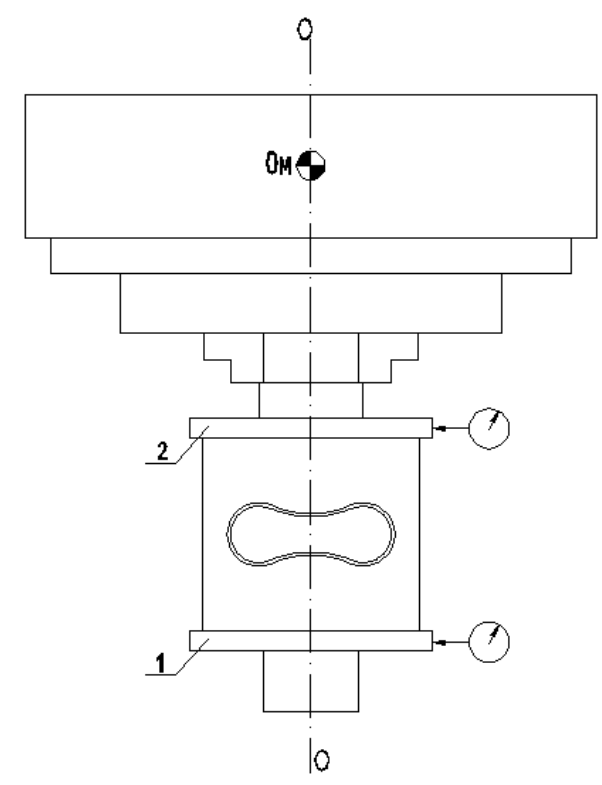

Fig. 5. Measuring radial beating with an indicator

For the surfaces to which the radial beating is taken, the two supporting boards 1 and 2 are selected. Measurement was performed at $30^{\circ} \mathrm{C}$ on axis $\mathrm{C}$. In Table 1 shows the measurement results.

TABLE I.

\begin{tabular}{|c|c|c|}
\hline \multirow{2}{*}{$\begin{array}{c}\text { Angle of rotation } \\
\left.\text { on axis C, }{ }^{\circ}\right]\end{array}$} & \multicolumn{2}{|c|}{ Radial beating, mm } \\
\cline { 2 - 3 } & Supporting board 1 & Supporting board 2 \\
\hline 0 & 0 & -0.06 \\
\hline 30 & 0.05 & -0.08 \\
\hline 60 & 0.1 & -0.1 \\
\hline 90 & 0.13 & -0.09 \\
\hline 120 & 0.12 & -0.08 \\
\hline 150 & 0.09 & -0.06 \\
\hline 180 & 0.04 & 0.03 \\
\hline 210 & -0.02 & 0.05 \\
\hline 240 & -0.06 & 0.07 \\
\hline 270 & -0.08 & 0.07 \\
\hline 300 & -0.07 & 0.06 \\
\hline 330 & -0.04 & 0.03 \\
\hline 360 & 0 & -0.06 \\
\hline
\end{tabular}

With the measured radial beating values, a virtual error model in the PTC Creo CAD system has been developed to determine how the axis of the work surface is displaced relative to the real axis of rotation of axis $\mathrm{C}$ (Figure 6).

The center of the supporting board 1 is constructed by passing a straight line connecting two points corresponding to the maximum and minimum radial beating. The center point is the center of the circle with a radius equal to the radius of the tool (supporting boards). 


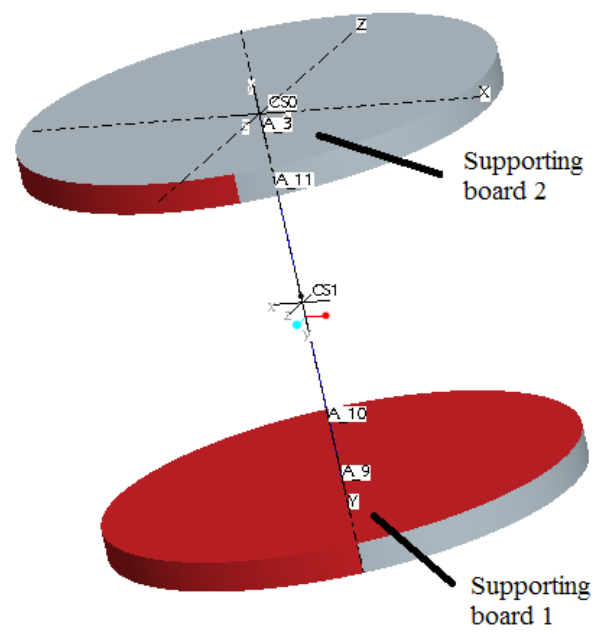

Fig. 6. Beating the workpiece to the real axis of the machine

Similarly, the center of the circle for the supporting board 2 is built. The red color of the supporting board shows the actual $\mathrm{C}$ axis position on the machine, and the gray shows the beating of the workpiece relative to the same axis. Once the two centers have been built, a line is drawn to connect them. The angle between the two axes, which is $0.02^{\circ}\left(0^{\circ} 1^{\prime} 12^{\prime \prime}\right)$, was measured with the toolbox. In Figure 7 shows the displacement of the workpiece axis due to the beating for the supporting boards 1 and 2 .
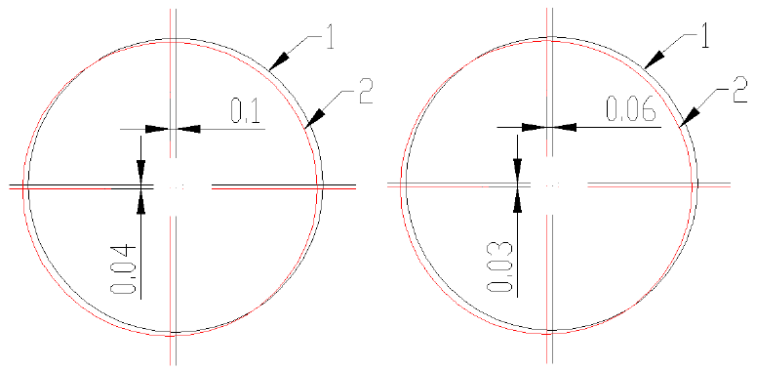

Fig. 7. Displacement of the workpiece axis due to the beating for the supporting boards 1 and 2

After the tool is centered (radial bevel reduction) with the chuck and flange assembly with the bolt system, the radial bevel is reduced to $0.06 \mathrm{~mm}$ for the supporting board 1 and $0.04 \mathrm{~mm}$ for the supporting board 2. After repositioning the tool on the side surface on an instrumental microscope, the dimension $b$ of the cutting track for the three cutting edges located at a central angle of $120^{\circ}$ in the sections shown in Figure 8.

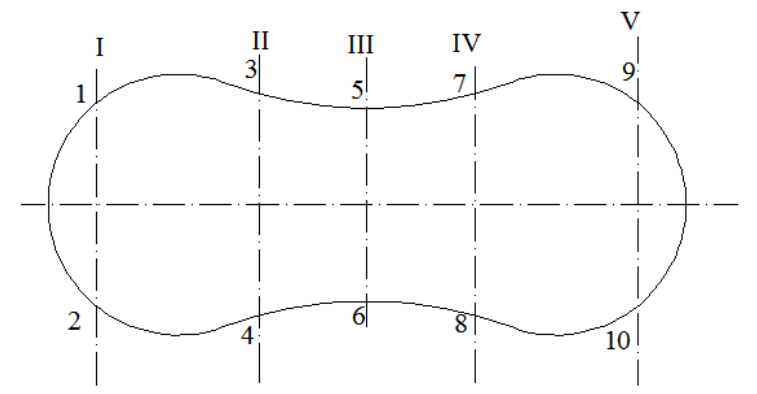

Fig. 8. Determination of the size $b$ in different sections of the cutting contour

Table 2 gives the measured values of the $b$ dimension of the cutting track.
TABLE II.

\begin{tabular}{|c|c|c|c|c|c|c|c|c|c|c|c|}
\hline \multirow{2}{*}{ № } & ๑ี & \multicolumn{2}{|c|}{ I } & \multicolumn{2}{|c|}{ II } & \multicolumn{2}{|c|}{ III } & \multicolumn{2}{|c|}{ IV } & \multicolumn{2}{|c|}{$\mathbf{V}$} \\
\hline & $\stackrel{\Xi}{0}$ & 1 & 2 & 3 & 4 & 5 & 6 & 7 & 8 & 9 & 10 \\
\hline 1 & & 0.4 & 0.3 & 0.35 & 0.25 & 0.3 & 0.3 & 0.3 & 0.3 & 0.3 & 0.45 \\
\hline 2 & & 0.3 & 0.3 & 0.25 & 0.2 & 0.3 & 0.2 & 0.4 & 0.2 & 0.35 & 0.3 \\
\hline 3 & & 0.4 & 0.4 & 0.3 & 0.3 & 0.3 & 0.3 & 0.3 & 0.3 & 0.4 & 0.3 \\
\hline
\end{tabular}

\section{CONCLUSION}

From Table 2, it can be seen that at $0.06 \mathrm{~mm}$ for supporting board 1 and $0.04 \mathrm{~mm}$ for supporting board 2, the error that occurs on the width of the cutting track is within $0.2 \mathrm{~mm}$. The highest accuracy is obtained by processing the second loop where a relatively uniform width of the cutting track is obtained. The results obtained are acceptable to a certain extent, but this necessitates subsequent finishing in order to achieve a uniform width of the cutting track.

\section{REFERENCES}

[1] Angel Lengerov, Galina Nikolcheva, Julieta Kaleycheva, Lyubomir Lazov, Statistical control of accuracy at machining ,Vide. Tehnologija. Resursi-Environment, Technology, Resources 3, pp. 183-187, 2017;

[2] Erik L.J. Bohez, Compensating for systematic errors in 5-axis NC machining, Computer-Aided Design, Volume 34, Issue 5, pages 347-420, 2002, DOI 10.1016/S0010-4485(01)00111-7;

[3] Fanuc Series 30i/31i/32i-Model B, Operator Manuals for machining center system;

[4] Hadzhiyski P., Knives for rotary dies with variable geometry, Journal "Engineering and automations problems", 2011, p. 131135, ISSN 0234-6206;

[5] Wahid Khan, Abdul \& Chen, Wuyi, A methodology for systematic geometric error compensation in five-axis machine tools. The International Journal of Advanced Manufacturing Technology, 2011, DOI: 10.1007/s00170-010-2848-3 